\title{
Neumonía en lactantes de 6 meses de edad y menores
}

\section{Pneumonia in infants 6 months of age and younger}

\author{
Dolores Lovera', Sara Amarilla, ${ }^{1,2}$, Fernando Galeano ${ }^{1,2}$, Celia Martínez de Cuellar ${ }^{1,2}$, Silvio \\ Apodaca', Antonio Arbo ${ }^{1,2}$
}

\section{RESUMEN}

Introducción: Poca atención se ha dado en literatura a la neumonía adquirida en la comunidad (NAC) en niños menores de 6 meses. Objetivos: Identificar los casos de NAC en pacientes de hasta 6 meses de edad, analizar las características demográficas, clínicas y evolutivas, y determinar los factores asociados a severidad. Materiales y Métodos: Se incluyeron a pacientes con NAC de 6 meses o menos de edad hospitalizados entre los años 2008 y 2019. Se definió como NAC a la presencia de síntomas y signos respiratorios (fiebre, tos y/o dificultad respiratoria) y evidencia en la radiografía de tórax de imagen de condensación. Se analizó las características demográficas, clínicas y factores asociados a severidad. Resultados: De 1582 casos de NAC en el periodo de estudio, se identificaron 256 casos en aquellos de 6 meses o menores. El $23 \%$ de los pacientes padecieron una comorbilidad. Hubo aislamiento microbiano en el $8 \%$ de los casos, siendo el Staphylococcus aureus y el Streptococcus pneumoniae los más frecuentes. Requirieron admisión en unidad de cuidados intensivos (UCI) 122 pacientes (48\%) de los cuales $88(72 \%)$ requirieron ventilación mecánica. Veintisiete pacientes $(10.5 \%)$ fallecieron. De 94 pacientes menores de 3 meses, 77 (82\%) se hospitalizaron en UCI en tanto que de los que no lo requirieron la mayoría fue mayor de 3 meses ( $p<0.001$; OR 2.61, 95\%IC 1.57-4.33). Conclusiones: El presente estudio muestra la severidad de las neumonías en lactantes de 6 meses o menores. La proporción de pacientes que requieren hospitalización en UCI es elevada, al igual que la mortalidad.

Palabras claves: Neumonía adquirida en la comunidad, niños $\leq 6$ meses, unidad de cuidados intensivos.

\begin{abstract}
Introduction: Little attention has been given in the literature to community acquired pneumonia (CAP) in children younger than 6 months. Objectives: To identify CAP cases in patients up to 6 months of age, analyze demographic, clinical and clinical course characteristics, and determine factors associated with severity. Materials and methods: Patients with CAP aged 6 months or younger, hospitalized between 2008 and 2019, were included. CAP was defined as the presence of respiratory symptoms and signs (fever, cough and / or respiratory distress) and evidence on chest radiography. of lung condensation. Demographic, clinical characteristics and factors associated with severity were analyzed. Results: Of 1582 CAP cases in the study period, 256 cases were identified in patients 6 months or younger. $23 \%$ of patients had a comorbidity. There was microbial isolation in $8 \%$ of cases, with Staphylococcus aureus and Streptococcus pneumoniae being the most frequent. One-hundred twenty two patients $(48 \%)$ required admission to the intensive care unit (ICU), of whom 88 (72\%) required mechanical ventilation. Twenty-seven patients (10.5\%) died. Of 94 patients younger than 3 months, 77 (82\%) were hospitalized in the ICU, while the majority of those who did not require hospitalization were older than 3 months ( $\mathrm{p}<0.001$; OR 2.61, 95\% CI 1.57-4.33). Conclusions: This study describes the severity of pneumonia in infants 6 months and younger. The proportion of patients requiring ICU hospitalization is high, as is mortality.
\end{abstract}

Key words: Community acquired pneumonia, children $\leq 6$ months, intensive care unit.

${ }^{1}$ Instituto de Medicina Tropical. Asunción, Paraguay.

${ }^{2}$ Universidad Nacional de Asunción. Facultad de Ciencias Médicas. San Lorenzo, Paraguay.

Correspondencia: Antonio Arbo Correo: antonioarbo@hotmail.com

Conflicto de interés: Los autores declaran no poseer conflicto de interés.

Recibido: 02/06/2020 Aceptado:29/06/2020

Doi: https://doi.org/10.31698/ped.47022020007

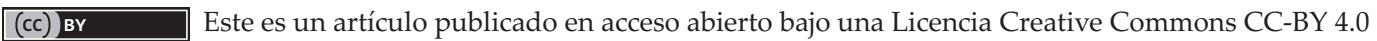




\section{INTRODUCCIÓN}

Las neumonías representan la principal causa de morbilidad y mortalidad en niños menores de 5 años a nivel del mundo ${ }^{(1)}$. Cada año fallecen alrededor de 10 millones de niños de este grupo de edad y aproximadamente 1 millón son a causa de neumonía ${ }^{(2)}$.

En Paraguay aproximadamente 1.000 niños menores de 5 años fallecen de neumonía cada año ${ }^{(3)}$. Si bien la literatura es importante en torno a las características de la neumonía en los niños menores de 5 años ${ }^{(4-6)}$, poca atención existe en la literatura a la presencia de neumonía en los niños menores de 6 meses de edad. Este grupo etario es particularmente susceptible a infecciones las cuales se acompañan de mayor afección en el huésped.

Los primeros seis meses de la vida representan el periodo de mayor vulnerabilidad en la vida de los individuos. Dentro de las infecciones, las neumonías son las más frecuentes después del mes de edad. La neumonía clínica infantil es causada por la exposición a factores de riesgo relacionados con el huésped, el medio ambiente y la infección. Factores de riesgo como la falta de lactancia materna exclusiva, bajo peso al nacer, desnutrición, contaminación del aire en interiores, hacinamiento y falta de adecuada inmunización se asocian con neumonía ${ }^{(7)}$. Aunque el uso universal de las vacunas conjugadas anti-Streptococcus pneumoniae y Haemophilus influenzae pueden tener un impacto en disminuir la incidencia de la neumonía bacteriana en niños ${ }^{(8)}$, los menores de 6 meses pueden no ser afectados todavía por el esquema de inmunización.

El objetivo del presente estudio fue describir los casos de neumonía en pacientes de 6 meses o menos de edad, analizar las características demográficas, clínicas y evolutivas, y determinar los factores asociados a severidad.

\section{MATERIALES Y MÉTODOS}

Sitio y población del estudio. El estudio se llevó a cabo en el Instituto de Medicina Tropical. Esta institución representa el principal centro de referencia de enfermedades infecciosas de la ciudad de Asunción, la capital del Paraguay. Se incluyeron en forma retrospectiva pacientes menores de 6 meses con diagnóstico de neumonía adquirida en la comunidad.

Criterios de definición. Se definió como neumonía adquirida en la comunidad a la presencia de síntomas y signos de neumonía (fiebre y síntomas respiratorios) y la evidencia en la radiografía de tórax de imagen de condensación, en un niño previamente sano, que fue adquirida en la comunidad. Niños que recibieron tratamiento antibiótico por más de 48 hs antes de la admisión hospitalaria fueron excluidos del estudio. Se incluyeron todos los casos de neumonía en niños de 6 meses o menores de edad hospitalizados entre enero 2008 a setiembre 2019. Variables. Se procedió a revisión de la biometría hemática, glicemia, función renal, hepática, electrolitos y gasometría. Además, se revisó en cada paciente el hemocultivo, y según el caso el cultivo de líquido pleural o aspirado traqueal. Pacientes con dificultad respiratoria (expresada por saturación de $\mathrm{O}_{2} \leq 92 \%$ con soporte de oxígeno en cánula nasal), tiraje universal, aleteo nasal o cianosis requirieron hospitalización en unidad de cuidados intensivos (UCI). Posteriormente se contrastaron las características de los pacientes que se hospitalizaron en UCI vs aquellos que no se hospitalizaron en UCI.

Análisis estadístico. Se utilizó la t de Student para el contraste de variables cuantitativas paramétricas y la chi cuadrada $\left(X^{2}\right)$ para el contraste de proporciones. Se realizó análisis univariado. Se calculó la razón de momios (OR). Se consideró significante una $\mathrm{p}<0.05$. Los análisis estadísticos se realizaron utilizando el paquete estadístico para ciencias sociales (SPSS versión 13, Chicago, IL, EE.UU.).

Ética. El estudio fue aprobado por el comité de ética de la institución. Dado que el estudio ha sido retrospectivo no se requirió el consentimiento de los padres o encargados, pero se aseguró el anonimato de la referido en el estudio. 


\section{RESULTADOS}

En el periodo de estudio se hospitalizaron en la institución 1582 casos de neumonía. De ellos 256 casos $(16 \%)$ fueron de 6 meses o menores. Un mil ochenta y siete pacientes (68.7\%) fueron mayores 6 meses y menores de 5 años, y 239 casos (15\%) fueron entre 5 años y 15 años.

La distribución etaria de los pacientes motivo del estudio se señala en la tabla 1. La distribución de niños fue homogénea en los diferentes meses y varió de 30 a 53 casos. En cuanto al sexo, el masculino fue más frecuente (152 pacientes) que el sexo femenino (104 pacientes) $(\mathrm{p}<0.05)$ (tabla 2$)$.

Tabla 1. Número y edad de los pacientes con neumonía hospitalizados.

\begin{tabular}{ccc}
\hline Edad & $\mathbf{n}=\mathbf{1 5 8 2}$ & Porcentaje $\%$ \\
\hline$\leq \mathbf{6}$ meses & 256 & 16.2 \\
$<1$ mes & 42 & 2.6 \\
2 meses & 52 & 3.2 \\
3 meses & 36 & 2.2 \\
4 meses & 30 & 1.9 \\
5 meses & 43 & 2.7 \\
6 meses & 53 & 3.3 \\
$<$ 1año & 478 & 30.2 \\
1 - 5 años & 865 & 54.7 \\
$>5$ años & 239 & 15.1 \\
\hline
\end{tabular}

Cincuenta y nueve pacientes (23\%) tuvieron una o más comorbilidades, de los cuales 19 pacientes (32\%) tuvieron desnutrición severa y $10(17 \%)$ hiperreactividad bronquial (tabla 2). La media de días de enfermedad antes del ingreso fue de 5.2 \pm 4.9 . La tos, fiebre y sibilancias fueron los síntomas más frecuentes observados (tabla 2). Distrés respiratorio moderado a severo se observó en 108 pacientes $(42 \%)$ y cianosis al ingreso se constató en 43 pacientes (17\%) (tabla 2$)$.

En cuanto a los datos laboratoriales, 115 pacientes (45\%) presentaron leucocitosis $\geq 15000 / \mathrm{mm}^{3}$ y se observó marcadores de inflamación (proteína C reactiva) positivos en 189 pacientes (74\%). Treinta y ocho pacientes $(15 \%)$ presentaron anemia (hemoglobina $<8 \mathrm{gr} / \mathrm{dl}$ ).

Los casos de neumonía presentaron frecuentemente complicaciones, de los cuales el choque, el derrame pleural y neumotórax fueron los más frecuentes (tabla 2). Ciento veintidós pacientes (48\%) requirieron admisión en unidad de cuidados intensivos, de los cuales 88 (72\%) requirieron ingreso a asistencia respiratoria mecánica (ARM). Veintisiete pacientes (10.5\%) fallecieron.

Tabla 2. Características demográficas y clínicas de pacientes con neumonía en niños $\leq 6$ meses

\begin{tabular}{|c|c|c|}
\hline & $N^{\circ}$ de pacientes & Porcentaje \\
\hline Número & 256 & 100 \\
\hline Sexo masculino & 152 & 59 \\
\hline Comorbilidad & 59 & 23 \\
\hline Desnutrición severa & 19 & 7 \\
\hline Hiperreactividad bronquial & 10 & 4 \\
\hline Prematurez & 6 & 2.3 \\
\hline Infección por VIH & 6 & 2.3 \\
\hline Cardiopatía congénita & 6 & 2.3 \\
\hline Síndrome de Down & 5 & 2 \\
\hline Hipotiroidismo & 4 & 1.6 \\
\hline Reflujo gastroesofágico & 2 & 0.8 \\
\hline Misceláneas & 15 & 5.9 \\
\hline \multicolumn{3}{|l|}{ Características clínicas } \\
\hline Días de enfermedad al ingreso & $5.2 \pm 4.9$ & \\
\hline Fiebre $\left(\mathrm{T}^{\circ} \mathrm{Ax}>37.5^{\circ} \mathrm{C}\right)$ & 186 & 72.7 \\
\hline Tos & 245 & 95.7 \\
\hline Sibilancias & 175 & 68.4 \\
\hline Distres respiratorio moderado(severo & 108 & 42.2 \\
\hline Derrame pleural & 21 & 8.2 \\
\hline Neumotórax & 5 & 2.0 \\
\hline Choque & 52 & 20.3 \\
\hline Ingreso a UCI & 122 & 47.7 \\
\hline Requerimiento de ARM & 88 & 34.4 \\
\hline Bacteriemia & 15 & 5.9 \\
\hline Días de internación & $11 \pm 11$ & \\
\hline Óbitos & 27 & 10.5 \\
\hline
\end{tabular}

En 21 pacientes ( $8 \%)$ hubo aislamiento microbiológico, principalmente en sangre $(n=15)$, líquido pleural $(n=3)$ y en secreción traqueal $(n=2)$ (tabla 3). En un caso el aislamiento fue en sangre y liquido pleural. Staphylococcus aureus $(\mathrm{n}=10,47,6 \%)$ y Streptococcus pneumoniae $(\mathrm{n}=5,23.8 \%)$ fueron los principales agentes etiológicos identificados.

Los pacientes hospitalizados en UCI fueron de menor edad que aquellos que no lo requirieron (tabla 4). Así, 77 pacientes (63\%) menores de 3 meses se hospitalizaron en UCI en tanto que de los que no lo requirieron la mayoría fue mayor de 3 meses (81 paciente, $60.4 \%$ ) ( $\mathrm{p}<0.001$; OR 2.61, 95\%IC 1.57-4.33). Los pacientes hospitalizados en UCI presentaron mas complicaciones como derrame pleural, neumotórax o choque, mientras que las mismas 
fueron mucho menos frecuentes en pacientes que no lo requirieron. En este sentido, la frecuencia de choque se observó en 51 pacientes de los 122 que se hospitalizaron en UCI en tanto en solo 1 de los 134 pacientes que no se hospitalizaron en $\mathrm{UCI}(\mathrm{p}<0.0001$; OR 95.53, 95\% IC 12.92-705.90), derrame pleural en 17 pacientes en los que se hospitalizaron en $\mathrm{UCI}$, en cambio en 4 de los que no se hospitalizaron ( $\mathrm{p}<0.01$; OR 5.26, 95\%IC 1.71-16.11), y neumotórax en 5 pacientes de los hospitalizados en UCI frente a ninguno de los que no se hospitalizaron $(\mathrm{p}=0.05$; OR 6.87, 95\%IC 0.81-57.98).
Tabla 3. Microbiología en casos de neumonía en niños $\leq 6$ meses

\begin{tabular}{lcc}
\hline Sitio de aislamiento & $\mathbf{N}^{\circ}$ de pacientes & Porcentaje \\
\hline Sangre & 15 & 71.4 \\
Liquido pleural & 3 & 14.3 \\
Secresión traqueal & 2 & 9.5 \\
Sangre y líquido pleural & 1 & 4.8 \\
Microorganismo & & \\
S. aureus & 10 & 47.6 \\
S. pneumoniae & 5 & 23.8 \\
P. aeruginosa & 2 & 9.5 \\
H. influenzaae tipo b & 1 & 4.8 \\
K. pneumoniae & 1 & 4.8 \\
Otros & 2 & 9.5 \\
\hline
\end{tabular}

Tabla 4. Características de niños con neumonía $\leq 6$ meses que ingresaron en UCI.

\begin{tabular}{lcccc}
\hline Variable & $\begin{array}{c}\text { UCI } \\
\mathbf{n = 1 2 2}\end{array}$ & $\begin{array}{c}\text { No UCI } \\
\mathbf{n = 1 3 4}\end{array}$ & $\mathbf{p}$ & OR (95\% IC) \\
\hline Sexo masculino & 75 & 77 & 0.59 & $1.18(0,71-1.94)$ \\
Edad $\leq 3 \mathrm{~m}$ & 77 & 53 & $<0.001$ & $2,61(1,57-4.33)$ \\
Derrame pleural & 17 & 4 & 0.003 & $5.26(1.71-16.11)$ \\
Neumotorax & 5 & 0 & $<0.05$ & No definido \\
Choque & 51 & 1 & $<0.001$ & $95.53(12.92-705.90)$ \\
ARM & 88 & 0 & $<0.001$ & No definido \\
Bacteremia & 12 & 3 & 0.02 & $4.76(1.31-17.3)$. \\
Hb $\leq 8$ & 33 & 9 & $<0.001$ & $5.14(2.34-11.29)$ \\
GB $>$ 15000/mm & 49 & 65 & 0.22 & $0.71(0.43-1.16)$ \\
Plaq $<150000 / \mathrm{mm}^{3}$ & 13 & 3 & $<0.01$ & $5.20(1.44-18.74)$ \\
Plaq $>$ 600000/mm & 3 & 26 & 0.87 & $0.91(0.48-1.71)$ \\
Según etiología & 22 & 26 & & \\
S. aureus & 9 & 1 & $<0.01$ & $10.59(1.32-84.89)$ \\
S. pneumonaie & 3 & 2 & 0.67 & $1.66(0.27-10.12)$ \\
Obitos & 26 & 1 & $<0.001$ & $36.02(4.80-270.05)$ \\
\hline
\end{tabular}

Aunque no hubo diferencias en el recuento de glóbulos blancos entre los pacientes que requirieron admisión en UCI con respecto a los que no lo requirieron, los primeros exhibieron una mayor frecuencia de cifra de plaquetas $<150000 / \mathrm{mm}^{3}$ (13 de 122 pacientes) en comparación a aquellos que no se hospitalizaron en UCI ( 3 de 133 pacientes) ( $\mathrm{p}<0.05$; OR 5.20, 95\%IC 1.44-18.74). La etiología por S. aureus fue claramente más frecuente en los pacientes que se hospitalizaron en UCI $(n=9)$ en comparación a aquellos que no requirieron $(\mathrm{n}=1)(\mathrm{p}<0.01 ;$ OR 10.59, $95 \%$ IC $1.32-84.89)$.

\section{DISCUSIÓN}

El presente estudio presenta una imagen de las características generales clínicas y laboratoriales de la neumonía en niños de 6 meses de edad o menores en un periodo de 12 años que se hospitalizaron en el
Instituto de Medicina Tropical. El principal hallazgo fue la elevada morbilidad y mortalidad de la neumonía en este grupo de edad.

La frecuencia fue homogénea en los diferentes meses de edad, aunque el sexo masculino fue el predominantemente afectado. La mayor afectación del sexo masculino puede explicarse en base genéticas $^{(9)}$, aun cuando no puede descartarse que la diferencia se explique en base a la tendencia que tienen las familias a mayor cuidado de los hijos varones. Aunque la prematurez, la hiperreatividad bronquial, la condición de VIH y la cardiopatía son hechos conocidos como predisponentes en este grupo etario a cuadros infecciosos, la condición de desnutrición fue algo relevante en la presente serie. La desnutrición es un condicionante de mayor susceptibilidad a infecciones ${ }^{(10)}$. 
Un aspecto importante de la serie fue la elevada morbilidad de los pacientes ${ }^{(11)}$. El $20 \%$ de los pacientes representaron choque e insuficiencia respiratoria el $34 \%$ de los casos. Esto explica la elevada proporción de pacientes hospitalizados en UCI (47.7\%). Esta cifra de hospitalización no es muy diferente a los hallado por la serie de Friz CQ y col ${ }^{(12)}$ y la de Lipsett SC y $\mathrm{col}^{(13)}$ donde la frecuencia de hospitalización de neumonía en UCI varió del 32\% al $38 \%$, pero es superior a lo reportado por Jain $\mathrm{S}$ y col ${ }^{(14)}$ en la que la frecuencia fue de $21 \%$.

Solo en el 8\% de los casos hubo aislamiento de un germen en sangre $u$ otro fluido. Esta frecuencia de detección, aunque baja es similar a reportes previos $^{(15,16)}$. Un aspecto importante de la serie fue el aislamiento de S.aureus como principal agente etiológico de neumonías en niños $\leq 6$ meses $(47.6 \%)$ seguido por S. pneumoniae (24\%). El hecho de aislamiento de $S$. aureus puede explicar en parte la severidad de los casos de neumonía en este grupo etario. De hecho, el aislamiento de $S$. aureus fue significativamente mayor en aquellos que se hospitalizaron en UCI $(\mathrm{p}<0.01$; OR $10-59,95 \%$ IC 1.32-84.89). Diferentes autores de diversas áreas geográficas revelan la patogenicidad del $S$. aureus ${ }^{(17,18)}$. Aunque la mortalidad de las infecciones por $S$. aureus comparado con estudios de la década de los $80^{(17)}$ ha disminuido en reportes de los últimos años ${ }^{(18)}$, todavía sigue observándose mortalidades elevadas en algunos centros hospitalarios ${ }^{(19)}$.

Un aspecto llamativo en esta serie fue la elevada frecuencia de hospitalización en UCI de los pacientes. Así, el $47.7 \%$ de los pacientes requirieron hospitalización en UCI, siendo la gran mayoría de los pacientes $(63 \%)$ menores de 3 meses. La asociación de mal pronóstico con la edad es algo reportado en la literatura ${ }^{(20)}$. Esta figura excede a lo reportado por otros autores donde la frecuencia de hospitalización en UCI de niños con neumonía es francamente menor ${ }^{(13,14)}$. Los pacientes hospitalizados en UCI exhibieron choque en el $42 \%$ de los casos. Esta cifra es más elevada de la observada en otras series, como en el estudio de Fritz CQ et al $^{(12)}$ donde la frecuencia de choque en niños que requirieron hospitalización fue del $30 \%$. De los datos laboratoriales, la frecuencia de trombocitopenia $<150000 / \mathrm{mm}^{3}$ y la anemia severa $(\mathrm{Hb} \leq 8 \mathrm{gr} / \mathrm{dl})$ fueron más frecuentes en aquellos pacientes que se hospitalizaron en UCI, lo cual ya fue observada en otros estudios ${ }^{(21)}$.

Los pacientes que fallecieron fueron los de menor edad ( $\leq 3$ meses), tuvieron neumonía grave (complicada con derrame pleural o neumotorax), ingresaron en choque o con insuficiencia respiratoria. Este cuadro clínico es característico de pacientes que se hospitalizaron en UCI en otras series ${ }^{(12,21)}$.

El presente estudio presenta limitaciones. Los casos son producto de análisis retrospectivo de casos que se hospitalizaron en un hospital de referencia por lo que estos resultados no pueden aplicarse directamente a casos vistos en hospitales de menor complejidad. Por otra parte, el papel de los virus no puede despreciarse, ya que los mismos se asocian a casos de neumonía en niños menores. Hay una superposición sustancial en las características clínicas y radiológicas de la bronquiolitis, enfermedad reactiva de las vías respiratorias y neumonía, en particular en niños pequeños. Incluso definiciones radiográficas estrictas no pueden distinguir entre estas entidades con precisión, lo que puede dar como resultado una posible clasificación errónea.

Finalmente, a pesar de estas limitaciones, nuestro estudio tuvo un gran tamaño de muestra de 256 pacientes estudiados durante una duración más larga de 12 años en un país de ingresos medianos bajos, lo que nos permitió acumular datos completos sobre la demografía, las características clínicas y la etiología niños hospitalizados con neumonía grave. 


\section{REFERENCIAS}

1. McAllister DA, Liu L, Shi T, Chu Y, Reed C, Burrows J, et al. Global, regional, and national estimates of pneumonia morbidity and mortality in children younger than 5 years between 2000 and 2015: a systematic analysis. Lancet Glob Health. 2019;7(1):e47-e57. doi: https://doi.org/10.1016/S2 214-109X(18)30408-X

2. Liu L, Oza S, Hogan D, Chu Y, Perin J, Zhu J, et al. Global, regional, and national causes of under-5 mortality in 2000-15: an updated systematic analysis with implications for the Sustainable Development Goals. Lancet. 2016; 388:3027-35. doi: https://doi.org/10.1016/S01406736(16)31593-8

3. Ministerio de Salud Pública y Bienestar Social. Indicadores básicos de salud Paraguay 2019[Internet]. Paraguay: MSPyBS; 2019 [Citado 18 may. 2020] Disponible en: http://portal.mspbs.gov.py/digies/wpcontent/uploads/2020/01/Indicadores-Basicos-de-SaludIBS-2019.pdf

4. Rambaud-Althaus C, Althaus F, Genton B, D'Acremont V. Clinical features for diagnosis of pneumonia in children younger than 5 years: a systematic review and metaanalysis. Lancet Infect Dis. 2015;15(4):439-50. doi: https://doi.org/10.1016/S1473-3099(15)70017-4

5. Ben-Shimol S, Givon-Lavi N, Grisaru-Soen G, Megged $\mathrm{O}$, Greenberg D, Dagan R, et al. Comparative incidence dynamics and serotypes of meningitis, bacteremic pneumonia and other-IPD in young children in the PCV era: Insights from Israeli surveillance studies. Vaccine. 2018;36(36):5477-5484. doi: https://doi.org/10.1016/j.vacci ne.2017.05.059

6. Araya S, Lovera D, Zarate C, Apodaca S, Acuña J, Sanabria G, et al. Application of a Prognostic Scale to Estimate the Mortality of Children Hospitalized with Community-acquired Pneumonia. Pediatr Infect Dis J. 2016;35(4):369-73. doi: https://doi.org/10.1097/INF.000000 0000001018

7. Rudan I, O'Brien KL, Nair H, Liu L, Theodoratou E, Qazi $\mathrm{S}$, et al. Epidemiology and etiology of childhood pneumonia in 2010: estimates of incidence, severe morbidity, mortality, underlying risk factors and causative pathogens for 192 countries. J Glob Health. 2013;3(1):010401. doi: https://doi.org/10.7189/jogh.03.010401

8 Peltola H. Hib and pneumococcal vaccines work also in far North. Acta Paediatr. 2019;108(10):1742-1743. doi: https://doi.org/10.1111/apa.14867

9. Alonso V, Fuster V, Luna F. Causes of neonatal mortality in Spain (1975-98): influence of sex, rural-urban residence and age at death. J Biosoc Sci. 2006;38(4):537-51. doi: https://doi.org/10.1017/S0021932005026957

10. DaVanzo J, Butz WP, Habicht JP. How biological and behavioural influences on mortality in Malaysia vary during the first year of life. Popul Stud. 1983; 37:381-402. doi: https://doi.org/10.1080/00324728.1983.10408868

11. Victora CG, Fuchs SC, Flores JA, Fonseca W, Kirkwood B. Risk factors for pneumonia among children in a Brazilian metropolitan area. Pediatrics 1994; 93:977-85.

12. Fritz CQ, Edwards KM, Self WH, Grijalva CG, Zhu Y, Arnold SR, et al. Prevalence, Risk Factors, and Outcomes of Bacteremic Pneumonia in Children. Pediatrics. 2019;144(1):e20183090. doi: https://doi.org/10.1542/ped s.2018-3090

13. Lipsett SC, Hall M, Ambroggio L, Desai S, Shah SS, Brogan TV, et al. Predictors of bacteremia in children hospitalized with community-acquired pneumonia. Hosp Pediatr. 2019;9:770-778. doi: https://doi.org/10.1542/hpe ds.2019-0149

14. Jain S, Williams DJ, Arnold SR, Ampofo K, Bramley $\mathrm{AM}$, Reed C, et al. Community-acquired pneumonia requiring hospitalization among U.S. children. N Engl J Med. 2015;372:835-845. doi: https://doi.org/10.1056/NEJM oa1405870

15. Murdoch DR, O'Brien KL, Driscoll AJ, Karron RA, Bhat N; Pneumonia Methods Working Group, et al. Laboratory methods for determining pneumonia etiology in children. Clin Infect Dis. 2012;54 Suppl 2:S146-52. doi: https://doi.org/10.1093/cid/cir1073

16. Shah SS, Dugan MH, Bell LM, Grundmeier RW, Florin TA, Hines EM, et al. Blood cultures in the emergency department evaluation of childhood pneumonia. Pediatr Infect Dis J. 2011;30(6):475-9. doi: https://doi.org/10.1097/ INF.0b013e31820a5adb

17. Chartrand SA, McCracken GH. Staphylococcal pneumonia in infants and children. Pediatr Infect Dis. 1982;1(1):19-23. doi: https://doi.org/10.1097/00006454198201000-00006

18. Moran GJ, Krishnadasan A, Gorwitz RJ, Fosheim GE, Albrecht V, Limbago B, et al. Prevalence of methicillinresistant staphylococcus aureus as an etiology of community-acquired pneumonia. Clin Infect Dis. 2012;54(8):1126-33. doi: https://doi.org/10.1093/cid/cis022. PMID: 22438343 
19. Gillet Y, Vanhems P, Lina G, Bes M, Vandenesch F, Floret $\mathrm{D}$, et al. Factors predicting mortality in necrotizing community-acquired pneumonia caused by Staphylococcus aureus containing Panton-Valentine leukocidin. Clin Infect Dis. 2007;45(3):315-21. doi: https://doi.org/10.1086/519263

20. Sehgal V, Sethi GR, Sachdev HPS, Satyanarayana L. Predictors of mortality in subjects hospitalized with acute lower respiratory tract infections. Indian Pediatr 1997;34:213-9.
21. Dembele BPP, Kamigaki T, Dapat C, Tamaki R, Saito M, Saito M, et al. Aetiology and risks factors associated with the fatal outcomes of childhood pneumonia among hospitalised children in the Philippines from 2008 to 2016: a case series study. BMJ Open. 2019;9(3):e026895. doi: https://doi.org/10.1136/bmjopen-2018-026895 